Pacific Journal of Mathematics

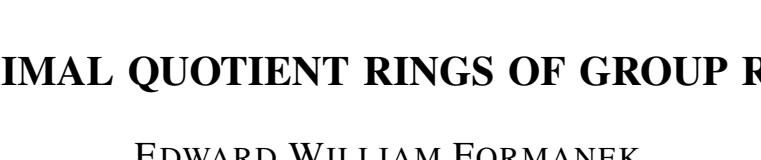




\section{MAXIMAL QUOTIENT RINGS OF GROUP RINGS}

\section{EDWARD FORMANEK}

Let $F[G]$ be the group ring of a group $G$ over a field $F$, and $\Delta$ the subgroup of $G$ consisting of those elements with only finitely many conjugates. Let $Q(R)$ denote the maximal (Utumi) quotient ring of a ring $R$. This paper proves: (1) If $H$ is a subnormal subgroup of $G, Q(F[H])$ is naturally embedded as a subring of $Q(F[G])$. (2) $Q(F[\Delta])$ contains the center of $Q(F[G])$. (3) If $F[G]$ is semiprime with center $C$, $Q(C)$ is the center of $Q(F[G])$. These results are analogues of theorems of M. Smith and D.S. Passman for the classical (Ore) quotient ring.

1. Introduction. Rings are associative and have a unit, and modules are unitary. Group rings will always be over fields, and we follow the definitions and notation of [5] for group rings and of [3] for quotient rings. In particular, if $F[G]$ is the group ring $G$ over $F$, then

$$
\begin{aligned}
& \Delta=\Delta(G)=\{g \in G: g \text { has finitely many conjugates }\} \\
& \Delta^{+}=\Delta^{+}(G)=\text { torsion subgroup of } G ; \\
& \theta: F[G] \rightarrow F[\Delta] \text { is the natural projection. }
\end{aligned}
$$

If $R$ is a ring, $Q=Q(R)$ is the maximal quotient ring of $R$.

There are many quotient rings which can be associated with a ring $R$. The two which have received the greatest attention are the classical (Ore) quotient ring and the maximal (Utumi) quotient ring. The classical quotient ring has a relatively straightforward description, but it is only defined for rings which satisfy the so-called Ore condition. In contrast the maximal quotient ring is less easy to describe but is defined for all rings. In both cases there are distinct notions of left and right quotient rings and we will always consider left quotient rings.

For group rings the classical quotient ring has been studied by Herstein and Small [2], Passman [5, 6], M. Smith [7], and P. F. Smith [8], and the maximal quotient ring has been studied by Burgess [1].

This paper investigates the relationship of the maximal quotient rings of group rings, subgroup rings, and the centers of group rings. The object is to obtain for the maximal quotient ring analogues of theorems of Passman and M. Smith on the classical quotient ring. Their techniques are used for the group ring arguments while the quotient ring arguments reflect the formalism of the maximal quotient ring. 
If $R$ is a subring of $S$, there is in general no relation between $Q(R)$ and $Q(S)$. Thus to say that $Q(R)$ is a subring of $Q(S)$ for a given $R$ and $S$ has little meaning unless accompanied by a precise interpretation, and this will be given in the body of the paper. Modulo this interpretation, the main results are summarized by the following theorem.

THEOREM. Let $F[G]$ be a group ring with center $C$.

(1) If $H$ is a subnormal subgroup of $G, Q(F[H])$ is a subring of $Q(F[G])$.

(2) $Q(F[\Delta])$ contains the center of $Q(F[G])$.

(3) If $F[G]$ is semiprime, $Q(C)$ is the center of $Q(F[G])$.

I do not know if the hypothesis that $F[G]$ be semiprime is required in (3). Passman [6] has proved the analogue of (3) for the classical quotient ring without this hypothesis.

2. Dense ideals and the maximal quotient ring. With each ring $R$ is associated a larger ring $Q=Q(R)$, called the maximal quotient ring of $R$. There are several equivalent constructions of $Q$. We will use the original one which is based on dense ideals and is due to Utumi [9, see 3, p. 96-99].

A left ideal $D$ of $R$ is dense if for each $a \in R$ the right annihilator of $D a^{-1}$ is zero, where $D a^{-1}=\{r \in R: r a \in D\}$. (Note that if $a$ is invertible, $D a^{-1}$ has the usual meaning.) Some of the basic properties of dense left ideals are (see [3, p. 96-98]):

(1) If $D_{1}$ is dense and $D_{1} \subseteq D_{2}$ then $D_{2}$ is dense.

(2) If $D$ is dense and $a \in R$, then $D a^{-1}$ is dense.

(3) If $D_{1}$ and $D_{2}$ are dense, so is $D_{1} \cap D_{2}$.

(4) If $D_{1}$ and $D_{2}$ are dense and $f: D_{2} \rightarrow R$ is a homomorphism, then $f^{-1}\left(D_{1}\right)$ is dense.

(5) If $R$ is commutative, $D$ is dense iff it has zero annihilator.

The maximal (left) quotient ring of $R$ is the set of all pairs $(f, D)$ where $D$ is a dense left ideal of $R$ and $f: D \rightarrow R$ is a homomorphism of left $R$-modules, modulo the equivalence relation $\left(f_{1}\right.$, $\left.D_{1}\right) \sim\left(f_{2}, D_{2}\right)$ if $f_{1}$ and $f_{2}$ agree on $D_{1} \cap D_{2}$. The sum and product of $\left(f_{1}, D_{1}\right)$ and $\left(f_{2}, D_{2}\right)$ are represented by the homomorphisms $f_{1}+$ $f_{2}: D_{1} \cap D_{2} \rightarrow R, f_{1} f_{2}: f_{2}^{-1}\left(D_{1}\right) \rightarrow R$. Each $a \in R$ defines a homomorphism $T_{a}: R \rightarrow R$ by $T_{a}(r)=r a$ and the map $a \mapsto T_{a}$ identifies $R$ with a subring of $Q$.

If $R$ is a subring of $S$, then $Q(R)$ is not in general a subring of $Q(S)$ and in general there is little relation between $Q(R)$ and $Q(S)$. However, there is a natural attempt to define a homomorphism $Q(R) \rightarrow Q(S)$ and when it succeeds it is automatically an injection 
of rings and then $Q(R)$ can be considered a subring of $Q(S)$. Namely, if $f: D \rightarrow R$ represents an element of $Q(R)$, one tries to extend $f$ to an $S$-homomorphism $f_{1}: S D \rightarrow S$. $f_{1}$ is unique if it exists but in general it does not exist. Even if $f_{1}$ exists, $S D$ may not be a dense ideal of $R$. If it happens that for every $(f, D) \in Q(R), S D$ is dense in $S$ and the extension $f_{1}$ exists, then $\left(f_{1}, S D\right) \in Q(S)$ and the map $(f, D) \rightarrow\left(f_{1}, S D\right)$ identifies $Q(R)$ with a subring of $Q(S)$. It turns out that this procedure works at least for some subrings of group rings.

\section{Dense ideals in group rings.}

THEOREM 1. Let $H$ be a normal subgroup of $G$. If $D$ is a dense ideal of $F[H]$, then $F[G] D$ is a dense left ideal of $F[G]$.

Proof. Let $a=a_{1} g_{1}+\cdots+a_{k} g_{k} \in F[G]$ where $a_{i} \in F, a_{i} \neq 0, g_{i} \in$ $G$. We have to show that the right annihilator of $(F[G] D) a^{-1}$ in $F[G]$ is zero. But for each $a_{\imath} g_{i}$

$$
(F[G] D)\left(a_{2} g_{i}\right)^{-1}=F[G] D g_{i}^{-1} \supseteqq g_{\imath} D g_{i}^{-1} \text {. }
$$

$D$ is dense in $F[H]$ so each $g_{i} D g_{i}^{-1}$ is dense in $F[H]$ since conjugation by $g_{i}$ is an automorphism of $F[H]$. Thus

$$
(F[G] D) a^{-1} \supseteqq \bigcap(F[G] D)\left(\alpha_{\imath} g_{2}\right)^{-1} \supseteqq \cap g_{i} D g_{i}^{-1}=J,
$$

where $J$ is dense in $F[H]$ since it is a finite intersection of dense left ideals of $F[H]$. $J$ has zero right annihilator in $F[H]$ so it has zero right annihilator in $F[G]$ since $F[G]$ is a free left $F[H]$-module. Hence $(F[G] D) a^{-1}$ also has zero right annihilator which shows that $F[G] D$ is dense.

REMARK. Theorem 1 is false if $H$ is not normal in $G$. For example, let $G$ be the free group generated by $g$ and $h$ and let $H$ be the subgroup generated by $h$. Then $D=F[H](h-1)$ is dense in $F[H]$ but $D^{\prime}=F[G] D=F[G](h-1)$ is not dense in $F[G]$. E.g. $D^{\prime}(g-1)^{-1}=0$ since $g-1$ and $h-1$ do not have a common left multiple. In this case $F[H]$ is a commutative domain and $Q(F[H])$ is just its classical quotient ring, a field. But no nonunit of $F[H]$ becomes invertible in $Q(F[G])$.

Assume now that $H$ is normal in $G$ and let $\left\{g_{i}\right\}$ be a set of coset representatives of $H$ in $G$. If $f: D \rightarrow F[H]$ represents an element of $Q(F[H])$, then $F[G] D={ }^{\oplus} g_{i} D$, a direct sum of abelian groups, so defining $\bar{f}: F[G] D \rightarrow F[G]$ by $\bar{f}\left(\sum g_{i} d_{i}\right)=\sum g_{i} f\left(d_{i}\right)$ gives a well-defined map. To verify that $\bar{f}$ is in fact $F[G]$-linear it suffices to show that 
if $a \in F[G], g_{i}$ is a coset representative, and $d \in D$, then $\bar{f}\left(a g_{i} d\right)=$ $a \bar{f}\left(g_{i} d\right)$. Letting $a g_{i}=\sum g_{j} a_{j}$, where $a_{j} \in F[H]$ (a finite sum), we have

$$
\begin{aligned}
\bar{f}\left(a g_{i} d\right) & =\bar{f}\left(\sum g_{j} a_{j} d\right)=\sum g_{j} \bar{f}\left(a_{j} d\right) \\
& =\sum g_{j} a_{j} f(d)=a g_{i} f(d)=a \bar{f}\left(g_{i} d\right) .
\end{aligned}
$$

It is clear that $f \rightarrow \bar{f}$ defines a ring monomorphism of $F[H]$ into $F[G]$ which is natural. We summarize this below, noting that it is enough for $H$ to be subnormal in $G$.

THEOREM 2. Let $H$ be a subnormal subgroup of $G$. Then $Q(F[H])$ is naturally identified with a subring of $Q(F[G])$ via the map ( $f$, $D) \rightarrow(\bar{f}, F[G] D)$, where $f: D \rightarrow F[H]$ represents an element of $Q(F[H])$.

From now on we will consider $Q(F[H])$ a subring of $Q(F[G])$ when Theorem 2 applies. If $G$ is abelian (or more generally, nilpotent) this means that $Q(F[G])$ contains $Q(F[H])$ for every subgroup $H$ of $G$.

4. The center of $Q(R)$. Suppose $f: D \rightarrow R$ represents a central element of $Q(R)$. Then $f$ commutes with the image of $R$ in $Q(R)$, namely with all the homomorphisms $T_{a}, a \in R$, where $T_{a}: R \rightarrow R$ is defined by $T_{a}(r)=r a$. $f T_{a}$ is defined on $T_{a}^{-1}(D)=D a^{-1}$ and $T_{a} f$ is defined on $D$. Since $f$ is central $f T_{a}$ and $T_{a} f$ agree on $D \cap D a^{-1}$. Hence for any $a \in R$ and $d \in D \cap D a^{-1}$

$$
f(d a)=f T_{a}(d)=T_{a} f(d)=f(d) a .
$$

Lemma 3. Suppose $f: D \rightarrow R$ represents a central element of $Q(R)$. Then $f$ can be extended to a map $f: D R \rightarrow R$ by

$$
f\left(d_{1} a_{1}+\cdots+d_{n} a_{n}\right)=f\left(d_{1}\right) a_{1}+\cdots+f\left(d_{n}\right) a_{n}
$$

for $d_{1}, \cdots, d_{n} \in D, a_{1}, \cdots, a_{n} \in R$. Hence every central element of $Q(R)$ is represented by a map $f: D \rightarrow R$ where $D$ is a two-sided ideal and $f$ is a homomorphism of $R$-bimodules-i.e., $f(r d s)=r f(d) s$ for $r, s \in$ $R, d \in D$.

Proof. The ony problem is to show that the extension of $f$ is well-defined-we must show that if $\sum d_{i} a_{i}=0$, then $\sum f\left(d_{1}\right) a_{i}=0$. Suppose $\sum d_{i} a_{i}=0$ and let $E$ be the dense left ideal $E=\bigcap D\left(d_{i} a_{i}\right)^{-1}$. If $b \in E$, then $b d_{i} \in D, b d_{i} a_{i} \in D$, so

$$
0=f\left(\sum b d_{i} a_{i}\right)=\sum f\left(b d_{i} a_{i}\right)=\sum f(b d) a_{i}=b \sum f\left(d_{i}\right) a_{i} .
$$

Hence $\sum f\left(d_{i}\right) a_{i}=0$, since it is a right annihilator of the dense left ideal $E$. 
Returning to group rings, the center of $F[G]$ is the set of finite sums $\sum x_{g} g$ which are constant on conjugacy classes and hence is a subring of $F[\Delta]$. Since $\Delta$ is normal in $F[G], Q(F[\Delta])$ is a subring of $Q(F[G])$ and it is reasonable to suppose that it contains the center of $Q(F[G])$. We will show this but first we need some preliminaries on $\theta: f[G] \rightarrow F[\Delta]$.

Let $\left\{g_{2}\right\}$ be a set of coset representatives of $\Delta$ in $G$, with $g_{1}=$ 1. If $a=a_{1}+g_{2} a_{2}+\cdots+g_{k} a_{k}$, where $a_{\imath} \in F[\Delta]$, then $\theta\left(g_{i}^{-1} a\right)=a_{i}$. From this the following lemma is routine (see [1, 4.5-4.6] for more general results).

Lemma 4. Let $D$ be a left ideal of $F[G]$. Then

(1) $\theta(D)$ is a left ideal of $F[\Delta]$.

(2) $F[G] \theta(D) \supseteqq D$.

(3) If $D$ is dense in $F[G], \theta(D)$ is dense in $F[\Delta]$.

(4) If $D$ is a two-sided ideal, so are $\theta(D)$ and $F(G) \theta(D)$.

The next result has had widespread use in the study of group rings.

Lemma 5. (M. Smith [7, Lemma 2.3], [5, Lemma 1.3]). Suppose $a, b, c, d \in F[G]$ and $a g b=$ cgd for all $g \in G$. Then $a \theta(b)=c \theta(d)$.

Lemma 6. Any central element of $Q(F[G])$ can be represented by a map $f: D \rightarrow F[G]$ where $D$ is a two-sided ideal of $F[G], \theta(D) \cong$ $D$, and $f(\theta(D)) \subseteq F[\Delta]$.

Proof. By Lemma 3, any central element can be represented by a bimodule homomorphism $f: D \rightarrow F[G]$, where $D$ is a two-sided ideal of $F[G]$, so we will be done if we can extend $f$ to a homomorphism $f_{1}: D_{1} \rightarrow F[G]$, where $D_{1}=F[G] \theta(D)$ and $f_{1}(\theta(D)) \subseteq F[\Delta]$.

Suppose $a \in D$, and let $a=a_{1}+g_{2} a_{2}+\cdots+g_{k} a_{k}, f(a)=b_{1}+g_{2} b_{2}+$ $\cdots+g_{k} b_{k}$ where $a_{\imath}, b_{i} \in F[\Delta]$, (possibly some $a_{i}, b_{i}$ are zero). $\theta(D)$ is the set of all such $a_{1}$, as $a$ varies over $D$, so if we can define $f_{1}: F[G] \theta(D) \rightarrow F[G]$ by $f_{1}\left(g a_{1}\right)=g b_{1}$ for any $g \in G$, this $f_{1}$ will be the required extension. The only difficulty is to verify that $f_{1}$ is welldefined-it will then automatically be an $F[G]$-module homomorphism, extend $f$, and map the dense idea. $\theta(D)$ of $F[\Delta]$ into $F[\Delta]$.

This amounts to showing that if $a_{1}=\theta(a)=0$, then $b_{1}=\theta(f(a))=$ 0 . To see this, suppose $\theta(\alpha)=0$ and let $d \in D$. Then for any $g \in G$ $d g f(a)=f(d g a)=f(d) g a$ since $f$ is a bimodule homomorphism.

$\therefore \quad d \theta(f(a))=f(d) \theta(a)=0$, by Lemma 5 .

$\therefore D \theta(f(a))=0$, so $\theta(f(a))=0$ since $D$ is dense in $F[G]$. 
Since $\Delta$ is normal in $G$, Theorem 2 says that $Q(F[\Delta])$ is (identified with) a subring of $Q(F[G])$. In the notation of Lemma $6, f_{2} \mid \theta(D)$ : $\theta(D) \rightarrow F[\Delta]$ is identified with $f_{1}: F[G] \theta(D) \rightarrow F[G]$ which represents the same element of $Q(F[G])$ as $f: D \rightarrow F[G]$. Thus we have shown:

THEOREM 7. The center of $Q(F[G])$ is a subring of $Q(F[\Delta])$.

5. Semiprime group rings. In this section, the following data is fixed. $F$ is a field, $G$ is a group, $\Delta=\Delta(G)$. We assume that $J^{+}(G)$ has no elements of order $p$ if $F$ has characteristic $p$. This is equivalent to assuming that $F[G]$ is semiprime by a theorem of Passman [5, Theorem 3.7]. It implies that $F[H]$ is semiprime whenever $H$ is a subgroup of $A$. Let $C$ denote the center of $F[G]$.

Passman used the following lemnas in his work on the classical quotient ring of group rings. It plays a similar role with respect to the maximal quotient ring. Because we have the additional hypothesis that $F[H]$ is semiprime we get the additional conclusion (over [6]) that $F[Z]^{-1} F[H]$ is semisimple.

Lemma 8. (Passman [6, Lemma 1]). Let $H \subseteq \Delta$ be a finitely generated normal subgroup of $G$. Then

(1) $H$ has a torsion-free central subgroup $Z$ of finite inder which is normal in $G$.

(2) The roing of fractions $F[R]^{-1} F[H]$ obtained by inverting the ronzero elements of the central domain $F[Q]$ is a finite-dimensional semisimple algebro over the field $F[Q]^{-1} F[Z]$.

IEMMA 9. Let $I \neq 0$ be a G-invariant ideal of F[A]. Then $I \cap C \neq 0$.

Proof. Let $H \subseteq \Delta$ be a finitely generated normal subgroup of $G$ with $I_{1}=I \cap F[H] \neq 0$, and let $Z$ be as in Lemama 8, $A=F[Z]^{-1} F[H]$, $J=A I_{1}$. $G$ acts on $A$ which is semisimple Artinian and $J$ is a $G$ invariant ideal of $A$, so $J$ is generated as an $A$-module by a $G$ invariant idempotent $e=a / b$, where $a \in I_{1}, b \in F[Z], b \neq 0$. Let $b_{1}=$ $b, b_{2}, \cdots, b_{n}$ be the finitely many $G$-conjugates of $b$. Then

$$
e=a / b=a b_{2} \cdots b_{n} / b_{1} \cdots b_{n} .
$$

$e$ and $b_{1} \cdots b_{n}$ are centralized by $G$, so $a b_{2} \cdots b_{n}$ is central in $F[G]$. Thus $0 \neq a b_{2} \cdots b_{n} \in I \cap C$, as required.

Lemma 10. Suppose $D$ is a dense ideal of $C$ and $f: D \rightarrow C$ is a C-homomorphism. Then

(1) $F[G] D$ is dense in $F[G]$. 
(2) $f$ has a unique extension to an $F[G]$-homomorphism

$$
\bar{f}: F[G] D \rightarrow F[G] .
$$

(3) $\bar{f}$ represents a central element of $Q(F[G])$.

Proof. (1) Since $D$ is central $(F[G] D) a^{-1} \supseteqq F[G] D$ for all $a \in$ $F[G]$ so to show that $F[G]$ is dense in $F[G]$ it suffices to show that $A=\operatorname{Ann}_{F[G]}(D)=0$. But if $A \neq 0$, Lemma 9 says that $A \cap C \neq 0$, which contradicts the hypothesis that $D$ is dense in $C$.

(2) If $f$ has such an extension $\bar{f}$ it is clearly unique and is defined by

$$
\bar{f}\left(a_{1} d_{1}+\cdots+a_{k} d_{k}\right)=a_{1} f\left(d_{1}\right)+\cdots+a_{k} f\left(d_{k}\right)
$$

for $a_{1}, \cdots, a_{k} \in F[G], d_{1}, \cdots, d_{k} \in D$.

The only problem is to show that $\bar{f}$ is well-defined-we must show that if $\sum a_{2} d_{\imath}=0$, then $\sum f\left(a_{\imath}\right) d_{\imath}=0$. Suppose $\sum a_{2} d_{i}=0$ and let $d \in D$. Since $d$ is central

$$
\begin{aligned}
d\left(\sum a_{i} f\left(d_{i}\right)\right) & =\sum a_{i} d f\left(d_{\imath}\right)=\sum a_{\imath} f\left(d d_{i}\right) \\
& =\sum a_{i} f\left(d_{\imath} d\right)=\left(\sum a_{\imath} d_{\imath}\right) f(d)=0 .
\end{aligned}
$$

Hence $\sum a_{\imath} f\left(d_{\imath}\right)=0$, since it annibilates the dense ideal $F[G] D$ on the right.

(3) First note that $\bar{f}: F[G] D \rightarrow F[G]$ is a bimodule homomorphism and suppose $g: D_{1} \rightarrow F[G]$ represents any element of $Q(F[G])$. Then for any $d \in D$ and $d_{1} \in D_{1}$,

$$
\bar{f} g\left(d d_{1}\right)=\bar{f}\left(d g\left(d_{1}\right)\right)=\bar{f}(d) g\left(d_{1}\right)=g\left(\bar{f}(d) d_{1}\right)=g \bar{f}\left(d d_{1}\right) .
$$

Thus $\bar{f} g$ and $g \bar{f}$ are defined and agree on $D D_{1}$. It is easy to see that $D D_{1}$ is a dense left ideal of $F[G]$, so $\bar{f} g$ and $g \bar{f}$ represent the same element of $G(F[G])$ and so $\bar{f}$ is central in $Q(F[G])$.

As in $\S 3,(f, D) \rightarrow(\bar{f}, F[G] D)$ is a ring momomorphism and we obtain a result analogous to Theorem 2.

THEOREM 11. Let $F[G]$ be a semiprime group ring with center $C$. Then $Q(C)$ is naturally identified with a central subring of $Q(F[G])$ via the $\operatorname{map}(f, D) \rightarrow(\bar{f}, F[G] D)$.

Now that we can consider $Q(C)$ a subring of the center of $Q(F[G])$ the final step is to show that it is the whole center. We already know that the center of $Q(F[H])$ is contained in $Q(F[\Delta])$, by Theorem 7 .

LemMa 12. If $d \in C, \mathrm{Ann}_{F[G]}(d)=F[G]$ e for some central idempotent $e$ of $F[G]$. 
Proof. Let $B=\operatorname{Ann}_{F[G]}(d), A=\operatorname{Ann}_{F[G]}(B) . \quad A$ is an annihilator ideal of $F[G]$ and $d \in A$ so a theorem of M. Smith [7, Corollary 5.6] [5, Theorem 25.4] says that there is a central idempotent $e$ of $F[G]$ such that $e \in A$ and $d e=d$. Then $B=\operatorname{Ann}_{F[G]}(d)=F[G](1-e)$.

Lemma 13. Let $D$ be a two-sided ideal of $F[\Delta]$ which is dense as a left ideal in $F[\Delta]$ and invariant under conjugation by elements of $G$. Then $D \cap C$ is dense in $C$.

Proof. Since $C$ is commutative, to show that $D \cap C$ is dense in $C$ it suffices to show that $\operatorname{Ann}_{c}(D \cap C)=0$. Let $c \in C, c \neq 0$. Then $\operatorname{Ann}_{F[G]}(c)=F[G] e$ for some central idempotent $e$ of $F[G]$ by Lemma 12. Since $D$ is dense, $D(1-e) \neq 0$ and by Lemma $9, D(1-e) \cap C$ contains a nonzero $d$. Now $d c \neq 0$ since otherwise we would have $d e=d, d(1-e)=d$, whence $d=0$.

Now consider a central element of $Q(F[G])$. By Lemma 6, it is represented by a map $f: D \rightarrow F[G]$ where $D$ is a two-sided ideal of $F[G], \theta(D) \subseteq D$, and $f(\theta(D)) \subseteq F[\Delta]$. By Theorem 7 and the remarks preceding, it lies in $Q(F[\Delta])$ where it is represented by $f \mid \theta(D): \theta(D) \rightarrow$ $F[\Delta] . \quad \theta(D)$ satisfies the hypothesis of Lemma 13 , so $\theta(D) \cap C$ is dense in $C$. $f$ maps $D \cap C$ into $C$ since $f\left(g^{-1} d g\right)=g^{-1} f(d) g$ for all $d \in D$. Thus $f \mid(\theta(D) \cap C): \theta(D) \cap C \rightarrow C$ represents an element of $Q(C)$ and we have shown

THEOREM 14. Let $F[G]$ be a semiprime group ring with center C. Then $Q(C)$ is the center of $Q(F[G])$.

Acknowledgment. I am indebted to D. S. Passman for many improvements in the presentation.

\section{REFERENCES}

1. W. Burgess, Rings of quotients of group rings, Canad. J. Math., 21 (1969), 865875.

2. I. N. Herstein and L. Small, Rings of quotients of group algebras, J. Algebra, 19 (1971), 153-155.

3. J. Lambek, Lectures on Rings and Modules, Blaisdell, Waltham, Massachusetts, 1966.

4. B. H. Neumann, Groups with finite classes of conjugate elements, Proc. London Math. Soc., (3) 1 (1951), 178-187.

5. D. S. Passman, Infinite Group Rings, Dekker, New York, 1971.

6. - On the ring of quotients of a group ring, Proc. Amer. Math. Soc., 33 (1972), 221-225.

7. M. Smith, Group algebras, J. Algebra, 18 (1971), 477-499.

8. P. F. Smith, Localization in group rings, Proc. London Math. Soc., (3) 22 (1971), 69-90.

9. Y. Utumi, On quotient rings, Osaka Math. J., 8 (1956), 1-18.

Received June 13, 1973. Research supported by NRC (Canada) grant A7171. 


\section{PACIFIC JOURNAL OF MATHEMATICS}

\section{EDITORS}

RICHARD ARENS (Managing Editor)

University of California

Los Angeles, California 90024

R. A. Beaumont

University of Washington

Seattle, Washington 98105
J. DugundJI

Department of Mathematics

University of Southern California

Los Angeles, California 90007

D. Gilbarg and J. Milgram

Stanford University

Stanford, California 94305

\section{ASSOCIATE EDITORS}

E. F. BECKENBACH

B. H. NeUMANN

F. WOLF

K. YosHIDA

\section{SUPPORTING INSTITUTIONS}

UNIVERSITY OF BRITISH COLUMBIA

CALIFORNIA INSTITUTE OF TECHNOLOGY

UNIVERSITY OF CALIFORNIA

MONTANA STATE UNIVERSITY

UNIVERSITY OF NEVADA

NEW MEXICO STATE UNIVERSITY

OREGON STATE UNIVERSITY

UNIVERSITY OF OREGON

OSAKA UNIVERSITY
UNIVERSITY OF SOUTHERN CALIFORNIA

STANFORD UNIVERSITY

UNIVERSITY OF TOKYO

UNIVERSITY OF UTAH

WASHINGTON STATE UNIVERSITY

UNIVERSITY OF WASHINGTON

$\stackrel{*}{*} \stackrel{*}{*} \stackrel{*}{*}$ AMERICAN MATHEMATICAL SOCIETY




\section{Pacific Journal of Mathematics}

\section{Vol. 53, No. $1 \quad$ March, 1974}

Martin Bartelt, Strongly unique best approximates to a function on a set, and a finite

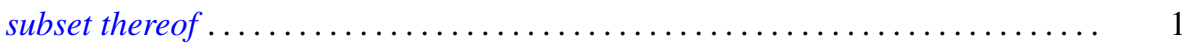

S. J. Bernau, Theorems of Korovkin type for $L_{p}$-spaces $\ldots \ldots \ldots \ldots \ldots \ldots \ldots \ldots \ldots$

S. J. Bernau and Howard E. Lacey, The range of a contractive projection on an

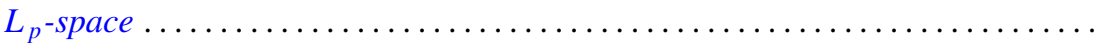

Marilyn Breen, Decomposition theorems for 3-convex subsets of the plane ......... Ronald Elroy Bruck, Jr., A common fixed point theorem for a commuting family of

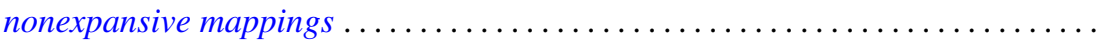

Aiden A. Bruen and J. C. Fisher, Blocking sets and complete $k$-arcs . . . . . . . 73

R. Creighton Buck, Approximation properties of vector valued functions . ......... 85

Mary Rodriguez Embry and Marvin Rosenblum, Spectra, tensor products, and

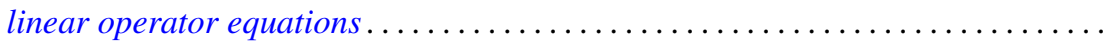

Edward William Formanek, Maximal quotient rings of group rings . . . . . . . . . 109

Barry J. Gardner, Some aspects of T-nilpotence . . . . . . . . . . . . . . . 117

Juan A. Gatica and William A. Kirk, A fixed point theorem for $k$-set-contractions

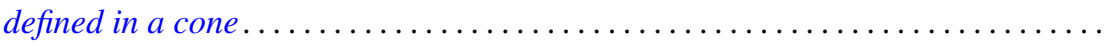

Kenneth R. Goodearl, Localization and splitting in hereditary noetherian prime

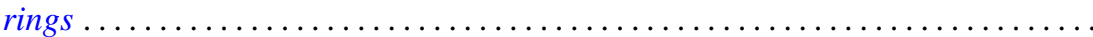

James Victor Herod, Generators for evolution systems with quasi continuous

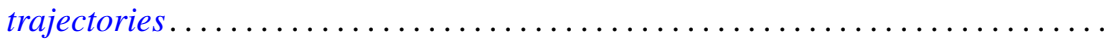

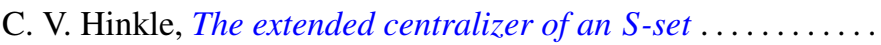

I. Martin (Irving) Isaacs, Lifting Brauer characters of p-solvable groups . . .

Bruce R. Johnson, Generalized Lerch zeta function ...........

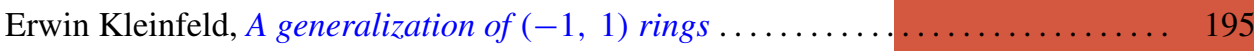

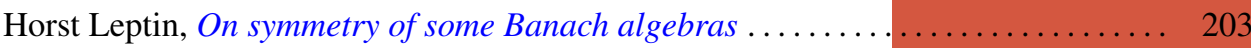

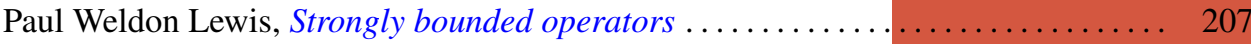

Arthur Larry Lieberman, Spectral distribution of the sum of self-adjoint

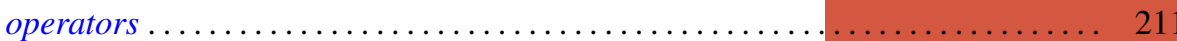

I. J. Maddox and Michael A. L. Willey, Continuous operators on paranormed spaces and matrix transformations

James Dolan Reid, On rings on groups ........................... 229

Richard Miles Schori and James Edward West, Hyperspaces of graphs are Hilbert cubes.

William H. Specht, A factorization theorem for p-constrained groups ...

Robert L Thele, Iterative techniques for approximation of fixed points of certain nonlinear mappings in Banach spaces ...............

Tim Eden Traynor, An elementary proof of the lifting theorem

Charles Irvin Vinsonhaler and William Jennings Wickless, Completely decomposable groups which admit only nilpotent multiplications .

Raymond O’Neil Wells, Jr, Comparison of de Rham and Dolbeault cohomology for

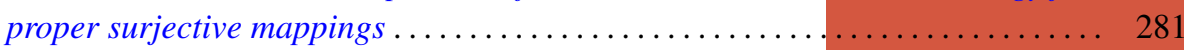

David Lee Wright, The non-minimality of induced central representations . . . . . 301 